\title{
Cascaded Convolutional Neural Network Architecture for Speech Emotion Recognition in Noisy Conditions
}

\author{
Youngja $\operatorname{Nam}^{1}$ and Chankyu Lee ${ }^{1,2, *}$ \\ 1 Humanities Research Institute, Chung-Ang University, Seoul 06974, Korea; btoj19@cau.ac.kr \\ 2 Department of Korean Language and Literature, Chung-Ang University, Seoul 06974, Korea \\ * Correspondence: leeck@cau.ac.kr
}

check for

updates

Citation: Nam, Y.; Lee, C. Cascaded Convolutional Neural Network Architecture for Speech Emotion Recognition in Noisy Conditions. Sensors 2021, 21, 4399. https:// doi.org/10.3390/s21134399

Academic Editor: Leon Rothkrantz

Received: 25 May 2021

Accepted: 24 June 2021

Published: 27 June 2021

Publisher's Note: MDPI stays neutral with regard to jurisdictional claims in published maps and institutional affiliations.

Copyright: (c) 2021 by the authors. Licensee MDPI, Basel, Switzerland. This article is an open access article distributed under the terms and conditions of the Creative Commons Attribution (CC BY) license (https:// creativecommons.org/licenses/by/ $4.0 /)$.

\begin{abstract}
Convolutional neural networks (CNNs) are a state-of-the-art technique for speech emotion recognition. However, CNNs have mostly been applied to noise-free emotional speech data, and limited evidence is available for their applicability in emotional speech denoising. In this study, a cascaded denoising CNN (DnCNN)-CNN architecture is proposed to classify emotions from Korean and German speech in noisy conditions. The proposed architecture consists of two stages. In the first stage, the DnCNN exploits the concept of residual learning to perform denoising; in the second stage, the CNN performs the classification. The classification results for real datasets show that the DnCNN$\mathrm{CNN}$ outperforms the baseline $\mathrm{CNN}$ in overall accuracy for both languages. For Korean speech, the DnCNN-CNN achieves an accuracy of $95.8 \%$, whereas the accuracy of the CNN is marginally lower (93.6\%). For German speech, the DnCNN-CNN has an overall accuracy of 59.3-76.6\%, whereas the CNN has an overall accuracy of $39.4-58.1 \%$. These results demonstrate the feasibility of applying the DnCNN with residual learning to speech denoising and the effectiveness of the CNN-based approach in speech emotion recognition. Our findings provide new insights into speech emotion recognition in adverse conditions and have implications for language-universal speech emotion recognition.
\end{abstract}

Keywords: cascaded DnCNN-CNN; speech emotion recognition; residual learning

\section{Introduction}

Emotions play a crucial role in social interactions as they provide important information about the thoughts and behavior of the speaker. Speech is one of the most efficient and fundamental ways of expressing emotion [1]. The study of speech emotion recognition, which aims to identify high-level affective contents of an utterance from low-level acoustic features, has drastically evolved over the past decade with the rapid development in human-machine interactions. Speech emotion recognition has a wide applicability, including in automated call centers [2], health care [3], onboard vehicle driving systems [4], education [5], and many other smart systems [6]. However, there is a long way to go to achieve a more natural interaction between humans and machines. In particular, determining the most effective features for emotion recognition remains an open issue, which makes speech emotion recognition very challenging. In fact, emotion classification is not straightforward, even for humans.

Speech emotion recognition systems consist of two main modules: feature extraction and classification. Feature extraction is the first core processing phase, in which the most relevant features that are available in the speech signal are extracted. Prior research on feature extraction is generally classified into four categories: continuous features, qualitative features, spectral features, and Teager energy operator (TEO)-based features [7] Continuous features include formant, timing, pitch-related features, and energy-related features [8]. Qualitative features are the perceptual correlates of the voice quality, e.g., being harsh, tense, and breathy [9]. Spectral features carrying information about the frequency contents that are present in speech are typically exploited as short-time representations of speech signals. Primary spectral features include linear predictor coefficients (LPC), linear 
predictor cepstral coefficients (LPCC), and mel-frequency cepstral coefficients (MFCC) [10]. TEO-based features, which are primarily employed in stress classification, include the TEOdecomposed FM variation, the normalized TEO autocorrelation envelope area, the criticalband-based TEO autocorrelation envelope area [11], and temporal feature integration for capturing the temporal dependency of successive feature observations [12].

Classification is the second core processing step in speech emotion recognition, in which the extracted features are mapped onto the relevant emotion classes. Previous studies have identified various classification algorithms to generate robust emotional features. Researchers have proposed support vector machines [13,14], Gaussian mixture models [15], hidden Markov models [16], artificial neural networks [17], the K-nearest neighbor [18], and binary decision trees [19]. These classifiers have demonstrated good emotion classification performance. However, these traditional approaches involve extracting well-established handcrafted features from signal cues, which typically requires significant human expertise and substantial effort. Furthermore, their performance varies widely across different speech databases.

Recently, deep learning has emerged as a promising research area in machine learning and has increasingly gained attention in various domains, including speech $[20,21]$ and image recognition [22]; natural language processing [23]; and, more recently, speech emotion recognition [24-26]. In particular, deep neural networks (DNNs) can learn high-level invariant features of the input signal from the raw data and provide a state-of-the-art classification performance [27,28]. Jiang et al. [29] proposed a hybrid DNN architecture to address the issue of heterogeneous acoustic features that usually degrade the classification performance in speech emotion recognition. This architecture focuses on extracting informative feature representations, which are fed into a fusion deep network, and then classified using a support vector machine. Recurrent neural networks (RNNs) can remember previous inputs in internal states and learn temporal contextual information; thus, they have shown improvements in terms of classifying emotion [30].

Convolutional neural networks (CNNs) [31] utilize a succession of layers of trainable convolution filters and optional pooling operations that are applied to local features. Although they were initially designed for computer vision tasks, CNNs have been efficient in recognition tasks of one-dimensional signals, such as audio [32] and speech [33]. In [34], one-dimensional CNN (1D CNN) and two-dimensional CNN (2D CNN) architectures outperformed standard feature-based classification and temporal feature integration methods in general audio classification. CNNs have also been effectively applied in speech emotion recognition tasks. Some studies have utilized CNN-based models trained on information generated from speech signals using spectrograms to extract high-level affective features [24,35]. A unique architecture based on a merged CNN, with a 1D CNN branch and a 2D CNN branch, was proposed to learn high-level features from raw input signals and log-mel spectrograms [36]. The 1D CNN and 2D CNN are first trained; then, their learned features are repurposed and transferred to the merged $\mathrm{CNN}$, which is subsequently fine-tuned. In another study [37], both spectrogram and phoneme embedding features were used as the input of a multichannel CNN model to achieve a good result on the interactive emotional dyadic motion capture (IEMOCAP) corpus. A lightweight and efficient $\mathrm{CNN}$-based architecture was also proposed to learn deep frequency features for speech emotion recognition [38]. In [39], a CNN-based architecture was tested on the acted emotional speech dynamic database (AESDD) by analyzing the sequential time frames and its performance surpassed some existing techniques, which rely on handcrafted features. Very recently, Abbaschian et al. [40] comprehensively and systematically reviewed major deep learning approaches employed in the speech emotion recognition research, combined with their associated speech databases. This review indicates that $\mathrm{CNN}$-based approaches play an important role in speech emotion recognition.

Therefore, CNNs have proven to be powerful for a range of speech emotion recognition tasks. In a real scenario, human speech emotion perception typically involves listening to the background noise. However, CNNs have been applied mostly on speech databases 
that are recorded in a controlled environment. Consequently, it remains unclear whether $\mathrm{CNNs}$ are effective for the recognition of emotions in noisy speech. Meanwhile, CNNs have proven to be successful in performing image denoising [41]. Very recently, a denoising CNN $(\mathrm{DnCNN})$ has shown promising results for removing environmental noise and enhancing the prediction accuracy to classify the defect patterns on semiconductor wafers [42]. To the best of our knowledge, DnCNN methods have not been evaluated in the speech domain. Furthermore, research on speech emotion recognition has focused on certain languages, such as English and German [43,44]. To date, no prior studies have investigated the effectiveness of deep learning architectures, including $\mathrm{CNN}$, in a Korean emotional speech database.

This study investigates the performance of a speech emotion recognition algorithm based on a DnCNN that is trained on a Korean emotional speech database. In particular, a cascaded DnCNN-CNN architecture was employed without using any traditional handcrafted features. For comparison, we investigated the performance of a baseline $\mathrm{CNN}$ model to further explore the capability of CNN-based architectures for speech signal denoising. The Korean database was recorded under less-controlled conditions and mixed with three types of environmental noise to simulate real-world listening situations. As the Korean database for this study contains a slight degree of noise, we also evaluated the proposed DnCNN-CNN alone on the original Korean database. This was done to further assess the effectiveness of the proposed classification method in recognizing emotions from noisy speech. In addition, we evaluated the proposed DnCNN-CNN on a popular German emotional speech database, EMO-DB. It was mixed with three types of environmental noise to further explore the feasibility of the DnCNN-CNN in emotion recognition for speech in another language. As for the Korean database, the CNN was also tested using the German speech database.

\section{Materials and Methods}

\subsection{Database}

We assessed the effectiveness of the proposed cascaded DnCNN-CNN architecture and the baseline $\mathrm{CNN}$ architecture using two speech emotional databases: the newly developed Chung-Ang database of Korean emotional speech (CADKES) and the Berlin Emotional Speech Database (EMO-DB) [44].

CADKES: Owing to the lack of an available Korean emotional speech dataset for this type of research, we created a dataset. Twenty-six actors (13 males and 13 females; mean age: 23.2 years; range: $19-27$ years) were individually recorded in a less-controlled condition at Chung-Ang University (CAU). The actors were recorded in the presence of background noise. All actors were undergraduates of the Department of Theatre at CAU and native Korean speakers. The recordings were made using a Rode USB microphone (Model Podcaster, RODE, Sydney, Australia), which was placed approximately $20 \mathrm{~cm}$ from each speaker. The computer had a 16-bit audio resolution, and the software program Praat [45] was used with a sampling rate of $44.1 \mathrm{kHz}$. The recordings were later down-sampled to $16 \mathrm{kHz}$. All actors produced 52 short sentences, which consisted of 40 declarative sentences and 12 interrogative sentences. The sentences contained a full set of Korean phonemes and were each uttered with five emotional intentions: neutral, happiness, sadness, anger, and fear. This resulted in 6760 sentences ( 26 actors $\times 52$ sentences $\times 5$ emotions). The Korean sentences were presented to 10 native Korean-speaking listeners for intellectual evaluation; all were determined to be intelligible. However, some recordings contained a slight level of ambient noise.

EMO-DB: The Berlin Emotional Speech Database (EMO-DB) in German is one of the most popular databases in speech emotion recognition. This database was collected from ten professional actors (five males) who were asked to express the given text (five short and five longer sentences) with seven emotions: neutral, happiness, sadness, anger, fear, disgust, and boredom. The recording occurred in an anechoic room at the Technical University of Berlin. A human perception test with 20 German listeners was conducted to ensure the 
emotional quality and naturalness of the recorded utterances. The EMO-DB consisted of 535 utterances that had an emotion recognition accuracy exceeding $80 \%$ in the perception test. Within the database, the seven emotions were not equally distributed. There were 79 neutral instances, 71 instances of happiness, 62 instances of sadness, 127 instances of anger, 69 instances of fear, 46 instances of disgust, and 81 instances of boredom.

\subsection{Noise Materials}

The present study was designed to evaluate the effectiveness of the DnCNN under a real-world noise environment. To simulate noise-corrupted speech signals, we employed the Diverse Environments Multichannel Acoustic Noise Database (DEMAND) [46], which provides a set of recordings of environmental noise in a variety of indoor and outdoor settings. The DEMAND recordings were performed with a 16-channel planar array of microphones and a target length of $5 \mathrm{~min}$. In this study, three types of indoor noise were selected: cafeteria noise recorded in a busy office cafeteria (PCAFETER), train station noise recorded in the main transfer area of a busy subway station (PSTATION), and subway noise recorded in a subway (TMETRO). Subsequently, the three types of noise were added to the utterances of CADKES and EMO-DB. The signal-to-noise ratio (SNR) was set to $10 \mathrm{~dB}$. Each speech database was mixed with the three types of environmental noise. This mix can be expressed as $z=(1-w) x+w y$, where $x$ is the original audio signal, $y$ is the environmental noise signal, and $\mathrm{w}$ is a weighting parameter.

\subsection{Spectrogram Generation}

A spectrogram is a $2 \mathrm{D}$ visual representation of the spectral density of an audio or speech signal (1D) as it varies with time at different frequencies. It has been demonstrated to be highly appropriate for converting a 1D speech-signal-based representation into a 2D CNN representation $[47,48]$. As the present study is concerned with learning highlevel features directly from speech signals using a 2D CNN-based architecture without considering handcrafted features, a spectrogram was used as input data to the 2D CNN.

The spectrogram was implemented using a short-term Fourier transform (STFT). The STFT first segments a long-time speech signal into overlapping shorter frames of equal length and then is separately applied on each frame. This reveals the Fourier spectrum on each frame. The Fourier transform provides not only the frequencies present in the signal, but also the magnitude of each frequency. In brief, the spectrogram is the output of the STFT, in which the horizontal axis represents the time, and the vertical axis represents the frequency of each frame. When considering the spectrogram, the darker the color is, the higher the magnitude at a particular time-frequency point is. The spectrogram has been used extensively in speech signal analysis, including speech emotion recognition. In this study, the spectrograms were extracted with a size of $256 \times 256$, and they had a $50 \%$ overlap.

\section{4. $\mathrm{CNN}$}

CNNs feature a multilayer feedforward architecture that typically consists of alternating convolutional and pooling layers followed by fully connected layers. The convolutional layer is a fundamental component of the CNN architecture, in which the feature extraction is performed by moving a predefined number of filters along the input. The pooling layer simplifies the output from the convolutional layer and achieves spatial invariance by down-sampling the resolution of the features. Pooling significantly reduces the computational parameters, thereby reducing the network computations. The most popular pooling algorithm is max pooling, which maintains the maximum value among the generated features and discards the others in the pooling region. The output feature maps of the pooling layer are connected to one or more fully connected layers, in which every input in one layer is connected to every output in another layer. The fully connected layer extracts the global features from the local convolutional features and performs classification on the extracted features. 


\subsection{Residual Learning}

The success of CNNs has been primarily attributed to the stacking of the distinct types of layers. The general concept behind their function is that the CNN layers progressively learn more abstract and complex features. Apparently, it is reasonable to assume that, as the network goes deeper, its accuracy improves. However, empirical evidence shows that with an increasing network depth, the accuracy becomes saturated or even degrades rapidly owing to the notorious vanishing gradient problem.

The residual learning framework was initially developed to address this performance degradation problem in image recognition [41]. Within the framework, the layers are reformulated for learning residual functions with respect to the layer inputs, rather than learning the unreferenced functions. The formulation of residual learning is as follows: $r=y-x$, where $y$ and $x$ are assumed to denote the underlying desired mapping and inputs to a few stacked layers, respectively. These stacked layers are hypothesized to approximate the residual function $r$, rather than $y$ [41]. Then, the original function becomes $\hat{x}=r+x$. The residual learning approach achieves a good performance for denoising tasks by effectively resolving the vanishing gradient and the degradation problem [49].

\subsection{Denoising $C N N$}

The concept of residual learning was subsequently adopted in the DnCNN for image denoising [50], which led to a significant increase in the advanced performance. The residual image learning strategy, $r=y-x$, fits the residual image $r$ more quickly than the clean image $x$ [51]. Subsequently, the modified residual learning strategy, $x=y+r$, where $r$ is targeted for our noise-removal method, is finally applied to the DnCNN to directly estimate the noise present in the input image.

Most recently, Chae and Bae [42] exploited a cascade of the DnCNN with residual learning and a CNN for identifying wafer map defects. These can be caused by dust in a cleanroom and/or by problems in the fabrication process or human error. The results showed that the residual-learning-based DnCNN outperformed the baseline CNN by $10 \%$. As an attempt to extend the cascaded DnCNN-CNN architecture to speech denoising, we can extend the concept of residual learning to emotional speech. Accordingly, we adopted the following loss function [52].

$$
l(\theta)=\frac{1}{2 N} \sum_{i=1}^{N}\left\|R\left(y_{i} ; \theta\right)-\left(y_{i}-x_{i}\right)\right\|^{2}
$$

where $N$ is the number of noisy speech patches and $\mathrm{R}\left(y_{i} ; \theta\right)$ is the residual mapping function. $\theta, y_{i}$, and $x_{i}$ denote the parameters of the proposed network, the noisy speech patch, and the clean speech patch, respectively.

\subsection{Network Architecture}

Figure 1 presents a block diagram of the proposed cascaded DnCNN-CNN architecture for emotional speech recognition. The cascaded architecture consists of two stages. The first stage is the denoising stage, in which the denoised spectrograms are generated by removing the noise from the noisy speech signals. Figure 2 shows the original and denoised sample spectrograms of an utterance in the Korean emotional speech database. The second stage is the classification stage, in which the CNN classifier recognizes the emotional states. Notably, this classifier serves as the baseline for comparison purposes. 


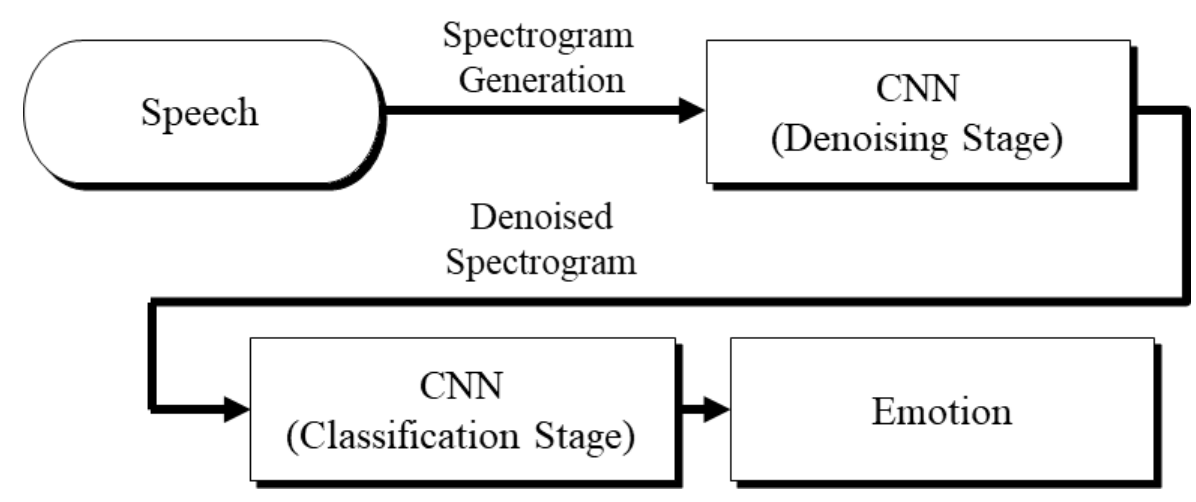

Figure 1. Block diagram of the proposed DnCNN-CNN architecture.

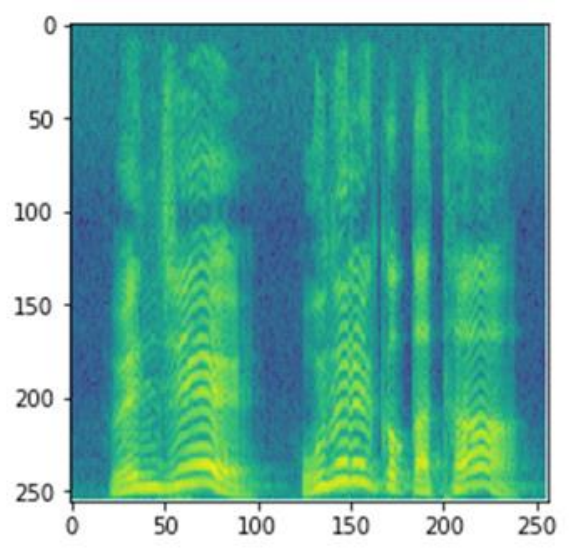

(a)

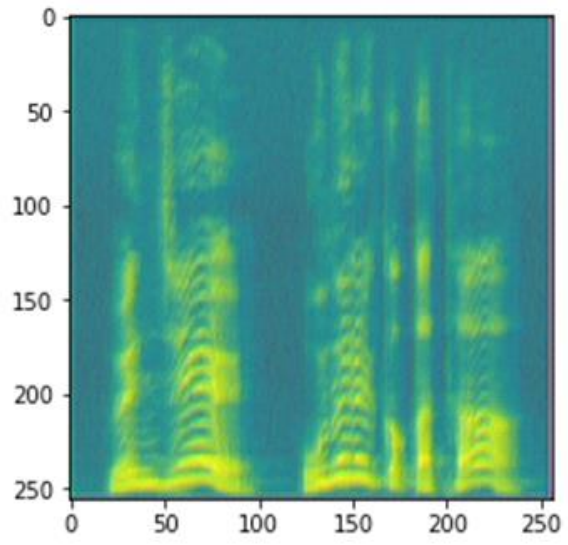

(b)

Figure 2. Sample spectrograms of an utterance in the Korean emotional speech database: (a) Original spectrogram; (b) Denoised spectrogram.

This study employed a network architecture that is similar to that used in a previous investigation [42]. Table 1 provides a detailed description of our proposed network architecture. The input of the network is a $256 \times 256$ spectrogram that is generated from noisy emotional speech signals. The residual learning stage consists of four convolutional layers. Batch normalization layers are used after the convolutional layers to normalize the inputs of each layer [53]. During this residual learning stage, denoised spectrograms are generated by removing the noise from the noisy speech signals.

The classification stage consists of four convolutional layers, four max pooling layers, one flattened layer, one dropout layer, and two fully connected layers. All convolutional layers have $32(3 \times 3)$ kernels, which are applied with a stride of 1 . Each convolutional layer is followed by a subsequent max pooling layer of $32(2 \times 2)$ kernels with a stride of 1 . Then, the output of the fourth max pooling layer is flattened to a single vector of size 8192 $\times 1$ before it is passed onto two fully connected layers with a $70 \%$ dropout. Subsequently, the output is fed into the first fully connected layer, which has 256 outputs. The second fully connected layer performs the five-class classification.

In this architecture, the rectified linear unit (ReLU) is used as the nonlinear activation function for the convolutional layers and the first fully connected layer, except for the second fully connected layer. The second fully connected layer uses the sigmoid function to squeeze the final output into the range from 0 to 1 . The network parameters were trained using the back-propagation algorithm with stochastic gradient descent. We trained the network for 1000 epochs at a learning rate of 0.01 . The dataset was randomly split into five subsets to perform a five-fold cross validation and the model with the best overall performance was selected for data analysis. 
Table 1. Structure of the proposed cascaded DnCNN-CNN.

\begin{tabular}{|c|c|c|c|}
\hline \multicolumn{4}{|c|}{ Denoising Stage } \\
\hline Layer Type & Output Size & Kernel Size & Stride \\
\hline $\begin{array}{l}\text { Convolutional Layer } 1 \\
\text { Batch Normalization }\end{array}$ & $256 \times 256 \times 64$ & $3 \times 3$ & $1 \times 1$ \\
\hline $\begin{array}{l}\text { Convolutional Layer } 2 \\
\text { Batch Normalization }\end{array}$ & $256 \times 256 \times 32$ & $3 \times 3$ & $1 \times 1$ \\
\hline $\begin{array}{c}\text { Convolutional Layer } 3 \\
\text { Batch Normalization }\end{array}$ & $256 \times 256 \times 16$ & $3 \times 3$ & $1 \times 1$ \\
\hline $\begin{array}{l}\text { Convolutional Layer } 4 \\
\text { Batch Normalization }\end{array}$ & $256 \times 256 \times 3$ & & \\
\hline \multicolumn{4}{|c|}{ Classification Stage } \\
\hline Convolutional Layer 1 & $256 \times 256 \times 32$ & $3 \times 3$ & $1 \times 1$ \\
\hline Max Pooling Layer 1 & $128 \times 128 \times 32$ & $2 \times 2$ & $1 \times 1$ \\
\hline Convolutional Layer 2 & $128 \times 128 \times 64$ & $3 \times 3$ & $1 \times 1$ \\
\hline Max Pooling Layer 2 & $64 \times 64 \times 64$ & $2 \times 2$ & $1 \times 1$ \\
\hline Convolutional Layer 3 & $64 \times 64 \times 128$ & $3 \times 3$ & $1 \times 1$ \\
\hline Max Pooling Layer 3 & $32 \times 32 \times 128$ & $2 \times 2$ & $1 \times 1$ \\
\hline Flattened Layer & 131,072 & - & - \\
\hline Dropout Layer & $70 \%$ & - & - \\
\hline Fully Connected Layer 1 & 256 & - & - \\
\hline Fully Connected Layer 2 & 5 & - & - \\
\hline
\end{tabular}

\section{Results}

The efficiency of the baseline CNN and the proposed cascaded DnCNN-CNN in speech emotion recognition under noisy environmental conditions was evaluated. Specifically, we evaluated the two classifiers on the Korean CADKES and German EMO-DB, which were mixed with PCAFETER, PSTATION, and TMETOR noise. As CADKES contains a slight level of noise, we further analyzed the classification performance of our proposed method on CADKES to comprehensively evaluate its efficiency. For comparison purposes, we also assessed the classification performance of the baseline CNN.

Tables 2 and 3 present the confusion matrices for the performance of the baseline $\mathrm{CNN}$ and the proposed DnCNN-CNN architecture, respectively, when CADKES was mixed with the PCAFETER noise. The results show that the baseline CNN classifier achieves the highest accuracy for sadness $(95.3 \%)$ and the lowest accuracy for fearful (36.1\%). The CNN has accuracies of $40.0 \%, 42.0 \%$, and $48.1 \%$ for anger, happiness, and neutral, respectively. Misclassification is the highest for the emotional state pair of fearful and sadness, in which fearful is misclassified as sadness $56.0 \%$ of the time. In addition, neutral $(47.9 \%)$, happiness $(43.0 \%)$, and anger $(37.7 \%)$ are most frequently misclassified as sadness. The proposed DnCNN-CNN architecture also achieves the highest accuracy for sadness $(92.7 \%)$, followed by neutral $(60.5 \%)$. The DnCNN-CNN classifier also exhibits the lowest accuracy for fearful (31.4\%); however, it exhibits accuracies of $47.2 \%$ and $49.2 \%$ for happiness and anger, respectively. Misclassification is the highest for the fearful-sadness pair $(55.2 \%)$, in which fearful is misclassified as sadness. Neutral is also most frequently misclassified as sadness (35.3\%). Happiness (34.4\%) and anger (29.4\%) are most frequently misclassified as sadness. The overall accuracy is 3.8\% higher for the DnCNN-CNN (56.1\%) than the CNN (52.3\%); however, the CNN performs better for the sadness and fearful emotions.

Tables 4 and 5 present the confusion matrices for the performance of the baseline CNN and the proposed DnCNN-CNN, respectively, when CADKES is mixed with PSTATION. The CNN classifier achieves the highest accuracy for sadness $(96.3 \%)$ and the lowest accuracy for happiness and fearful (37.0\%). The recognition accuracies of neutral and anger are $44.0 \%$ and $45.5 \%$, respectively. Misclassification is the highest for the fearful-sadness 
pair, where fearful is misclassified as sadness $57.0 \%$ of the time. This is followed by the neutral-sadness pair (52.5\%), happiness-sadness pair (45.3\%), and anger-sadness pair $(37.0 \%)$. The $\mathrm{CNN}$ achieves an overall accuracy of $51.9 \%$. The DnCNN-CNN also achieves the highest accuracy for sadness (92.6\%) and the lowest accuracy for happiness (33.9\%). The accuracies of neutral, anger, and fearful are $47.9 \%, 44.6 \%$, and $50.2 \%$, respectively. The DnCNN-CNN shows parallel misclassification patterns that are also observed for the $\mathrm{CNN}$; neutral $(46.1 \%)$, fearful $(42.4 \%)$, happiness $(36.3 \%)$, and anger $(30.3 \%)$ are most frequently misclassified as sadness. The DnCNN-CNN (53.9\%) achieves a $2 \%$ increase in the overall accuracy in comparison with the $\mathrm{CNN}(51.9 \%)$; however, the $\mathrm{CNN}$ performs better for the happiness, sadness, and anger emotions.

Table 2. Confusion matrix of the emotion classification results of the baseline CNN that was trained on the Korean CADKES mixed with the PCAFETER noise.

\section{Predicted Emotion}

\begin{tabular}{|c|c|c|c|c|c|c|}
\hline \multirow{6}{*}{ True emotion } & & Neutral & Happiness & Sadness & Anger & Fearful \\
\hline & Neutral & $48.1 \%$ & $0.7 \%$ & $47.9 \%$ & $1.7 \%$ & $1.5 \%$ \\
\hline & Happiness & $10.4 \%$ & $42.0 \%$ & $43.0 \%$ & $1.8 \%$ & $2.8 \%$ \\
\hline & Sadness & $2.2 \%$ & $0.7 \%$ & $95.3 \%$ & $0.5 \%$ & $1.3 \%$ \\
\hline & Anger & $9.2 \%$ & $6.6 \%$ & $37.7 \%$ & $40.0 \%$ & $6.6 \%$ \\
\hline & Fearful & $3.2 \%$ & $1.7 \%$ & $56.0 \%$ & $3.0 \%$ & $36.1 \%$ \\
\hline & & & Overall a & : 52.3\% & & \\
\hline
\end{tabular}

Table 3. Confusion matrix of the emotion classification results of the proposed cascaded DnCNN-CNN that was trained on the Korean CADKES mixed with the PCAFETER noise.

\section{Predicted Emotion}

\begin{tabular}{|c|c|c|c|c|c|c|}
\hline \multirow{6}{*}{ True emotion } & & Neutral & Happiness & Sadness & Anger & Fearful \\
\hline & Neutral & $60.5 \%$ & $0.8 \%$ & $35.3 \%$ & $2.8 \%$ & $0.6 \%$ \\
\hline & Happiness & $14.0 \%$ & $47.2 \%$ & $34.4 \%$ & $3.5 \%$ & $0.9 \%$ \\
\hline & Sadness & $3.7 \%$ & $0.8 \%$ & $92.7 \%$ & $1.9 \%$ & $0.9 \%$ \\
\hline & Anger & $11.8 \%$ & $7.0 \%$ & $29.4 \%$ & $49.2 \%$ & $2.7 \%$ \\
\hline & Fearful & $6.2 \%$ & $1.7 \%$ & $55.2 \%$ & $5.5 \%$ & $31.4 \%$ \\
\hline \multicolumn{7}{|c|}{ Overall accuracy: 56.1\% } \\
\hline
\end{tabular}

Table 4. Confusion matrix of the emotion classification results of the baseline CNN that was trained on the Korean CADKES mixed with the PSTATION noise.

\begin{tabular}{|c|c|c|c|c|c|c|}
\hline & \multicolumn{6}{|c|}{ Predicted Emotion } \\
\hline \multirow{7}{*}{ True emotion } & & Neutral & Happiness & Sadness & Anger & Fearful \\
\hline & Neutral & $44.0 \%$ & $0.1 \%$ & $52.5 \%$ & $1.9 \%$ & $1.5 \%$ \\
\hline & Happiness & $10.5 \%$ & $37.0 \%$ & $45.3 \%$ & $4.3 \%$ & $2.9 \%$ \\
\hline & Sadness & $1.3 \%$ & $0.3 \%$ & $96.3 \%$ & $0.2 \%$ & $1.9 \%$ \\
\hline & Anger & $8.5 \%$ & $5.6 \%$ & $37.0 \%$ & $45.5 \%$ & $3.4 \%$ \\
\hline & Fearful & $1.9 \%$ & $0.7 \%$ & $57.0 \%$ & $3.5 \%$ & $37.0 \%$ \\
\hline & \multicolumn{6}{|c|}{ Overall accuracy: 51.9\% } \\
\hline
\end{tabular}


Table 5. Confusion matrix of the emotion classification results of the proposed cascaded DnCNN-CNN that was trained on the Korean CADKES mixed with the PSTATION noise.

\begin{tabular}{|c|c|c|c|c|c|c|}
\hline & \multicolumn{6}{|c|}{ Predicted Emotion } \\
\hline \multirow{6}{*}{ True emotion } & & Neutral & Happiness & Sadness & Anger & Fearful \\
\hline & Neutral & $47.9 \%$ & $0.5 \%$ & $46.1 \%$ & $1.5 \%$ & $3.9 \%$ \\
\hline & Happiness & $17.0 \%$ & $33.9 \%$ & $36.3 \%$ & $5.3 \%$ & $7.5 \%$ \\
\hline & Sadness & $2.5 \%$ & $0.3 \%$ & $92.6 \%$ & $1.1 \%$ & $3.4 \%$ \\
\hline & Anger & $11.6 \%$ & $3.4 \%$ & $30.3 \%$ & $44.6 \%$ & $10.1 \%$ \\
\hline & Fearful & $3.6 \%$ & $0.7 \%$ & $42.4 \%$ & $3.0 \%$ & $50.2 \%$ \\
\hline
\end{tabular}

Tables 6 and 7 present the confusion matrices for the performance of the baseline CNN and the proposed DnCNN-CNN architecture, respectively, when the CADKES is mixed with the TMETRO noise. The results indicate that the CNN classifier exhibits the highest classification accuracy for sadness (93.8\%) and the lowest accuracy for anger (47.9\%). The neutral, happiness, and fearful emotions have an accuracy of 58.0\%, 48.6\%, and 51.7\%, respectively. Fearful (42.0\%), neutral (38.1\%), happiness (35.7\%), and anger (27.7\%) are most frequently misclassified as sadness. For the DnCNN classifier, sadness is the most accurately recognized emotion $(90.6 \%)$, whereas anger is the least accurately recognized emotion (50.4\%). Neutral, happiness, and fearful were recognized with accuracies of 79.2\%, $52.8 \%$, and $55.8 \%$, respectively. Fearful (35.5\%), happiness $(20.0 \%)$, neutral $(17.2 \%)$, and anger $(13.4 \%)$ are most frequently misclassified as sadness. The DnCNN-CNN $(64.8 \%)$ performs better, overall, than the $\mathrm{CNN}(60.1 \%)$ by $4.7 \%$. In addition, the proposed classifier performs better for all emotions except for the sadness emotion.

Table 6. Confusion matrix of the emotion classification results of the baseline CNN that was trained on the Korean CADKES mixed with the TMETRO noise.

\begin{tabular}{|c|c|c|c|c|c|c|}
\hline & \multicolumn{6}{|c|}{ Predicted Emotion } \\
\hline \multirow{6}{*}{ True emotion } & & Neutral & Happiness & Sadness & Anger & Fearful \\
\hline & Neutral & $58.0 \%$ & $0.7 \%$ & $38.1 \%$ & $0.5 \%$ & $2.6 \%$ \\
\hline & Happiness & $10.8 \%$ & $48.6 \%$ & $35.7 \%$ & $1.8 \%$ & $3.1 \%$ \\
\hline & Sadness & $1.8 \%$ & $0.5 \%$ & $93.8 \%$ & $0.7 \%$ & $3.1 \%$ \\
\hline & Anger & $10.5 \%$ & $8.2 \%$ & $27.7 \%$ & $47.9 \%$ & $5.7 \%$ \\
\hline & Fearful & $2.3 \%$ & $2.2 \%$ & $42.0 \%$ & $1.8 \%$ & $51.7 \%$ \\
\hline \multicolumn{7}{|c|}{ Overall accuracy: $60.1 \%$} \\
\hline
\end{tabular}

Table 7. Confusion matrix of the emotion classification results of the proposed cascaded DnCNN-CNN that was trained on the Korean CADKES mixed with the TMETRO noise.

\begin{tabular}{|c|c|c|c|c|c|c|}
\hline & \multicolumn{6}{|c|}{ Predicted Emotion } \\
\hline \multirow{6}{*}{ True emotion } & & Neutral & Happiness & Sadness & Anger & Fearful \\
\hline & Neutral & $79.2 \%$ & $1.4 \%$ & $17.2 \%$ & $0.3 \%$ & $1.9 \%$ \\
\hline & Happiness & $20.3 \%$ & $52.8 \%$ & $20.0 \%$ & $1.4 \%$ & $5.6 \%$ \\
\hline & Sadness & $4.2 \%$ & $0.2 \%$ & $90.6 \%$ & $0.7 \%$ & $4.3 \%$ \\
\hline & Anger & $17.0 \%$ & $10.7 \%$ & $13.4 \%$ & $50.4 \%$ & $8.6 \%$ \\
\hline & Fearful & $5.7 \%$ & $2.0 \%$ & $35.5 \%$ & $1.0 \%$ & $55.8 \%$ \\
\hline & \multicolumn{6}{|c|}{ Overall accuracy: $64.8 \%$} \\
\hline
\end{tabular}


As mentioned above, we evaluated the performances of the baseline $\mathrm{CNN}$ and the proposed cascaded DnCNN-CNN on the original CADKES that contains a slight level of noise to examine whether the classifiers perform differently. Tables 8 and 9 provide the emotion classification results of the $\mathrm{CNN}$ and the $\mathrm{DnCNN}-\mathrm{CNN}$, respectively. The results show that both classifiers have an excellent performance on the original CADKES. The $\mathrm{CNN}$ classifier yields an overall accuracy of $93.6 \%$. All the emotion classes are recognized with an accuracy above $90 \%$. The best recognized emotion is neutral (95.4\%). Meanwhile, misclassification is the highest for the sadness-fearful pair, where sadness is misclassified as fearful $3.8 \%$ of the time. This is followed by the sadness-neutral pair $(3.6 \%)$ and the anger-happiness pairs (3.6\%). The DnCNN-CNN architecture achieves a high overall accuracy of $95.8 \%$. Neutral, sadness, and anger $(>96 \%)$ have a relatively higher classification accuracy than happiness $(94.7 \%)$ and fearful $(93.0 \%)$. Misclassification is the highest for the emotional state pair of happiness and anger, where happiness is misclassified as anger $3.7 \%$ of the time. These results indicate that the proposed DnCNN-CNN classifier outperforms the baseline $\mathrm{CNN}$ classifier in terms of the overall accuracy. In addition, the proposed classifier performs better than the baseline classifier in terms of the accuracy for every class of emotion. The highest improvement in the classification accuracy is observed for sadness $(6.4 \%)$, whereas the lowest improvement in the classification accuracy is observed for happiness $(0.5 \%)$.

Table 8. Confusion matrix of the emotion classification results of the baseline CNN that was trained on the original Korean CADKES.

\begin{tabular}{|c|c|c|c|c|c|c|}
\hline & \multicolumn{6}{|c|}{ Predicted Emotion } \\
\hline \multirow{6}{*}{ True emotion } & & Neutral & Happiness & Sadness & Anger & Fearful \\
\hline & Neutral & $95.4 \%$ & $1.4 \%$ & $2.2 \%$ & $0.3 \%$ & $0.7 \%$ \\
\hline & Happiness & $2.1 \%$ & $94.2 \%$ & $0.8 \%$ & $1.9 \%$ & $1.0 \%$ \\
\hline & Sadness & $3.6 \%$ & $1.6 \%$ & $90.7 \%$ & $0.4 \%$ & $3.8 \%$ \\
\hline & Anger & $0.9 \%$ & $3.6 \%$ & $0.6 \%$ & $93.9 \%$ & $1.1 \%$ \\
\hline & Fearful & $2.1 \%$ & $1.8 \%$ & $3.2 \%$ & $0.8 \%$ & $92.1 \%$ \\
\hline \multicolumn{7}{|c|}{ Overall accuracy: $93.6 \%$} \\
\hline
\end{tabular}

Table 9. Confusion matrix of the emotion classification results of the proposed cascaded DnCNN-CNN that was trained on the original Korean CADKES.

\begin{tabular}{|c|c|c|c|c|c|c|}
\hline & \multicolumn{6}{|c|}{ Predicted Emotion } \\
\hline \multirow{7}{*}{ True emotion } & & Neutral & Happiness & Sadness & Anger & Fearful \\
\hline & Neutral & $98.1 \%$ & $0.9 \%$ & $1.0 \%$ & $0.0 \%$ & $0.0 \%$ \\
\hline & Happiness & $1.0 \%$ & $94.7 \%$ & $0.6 \%$ & $3.7 \%$ & $0.0 \%$ \\
\hline & Sadness & $1.2 \%$ & $0.6 \%$ & $97.1 \%$ & $0.0 \%$ & $1.2 \%$ \\
\hline & Anger & $0.5 \%$ & $1.1 \%$ & $1.1 \%$ & $96.2 \%$ & $1.1 \%$ \\
\hline & Fearful & $1.9 \%$ & $1.4 \%$ & $1.9 \%$ & $1.7 \%$ & $93.0 \%$ \\
\hline & \multicolumn{6}{|c|}{ Overall accuracy: 95.8\% } \\
\hline
\end{tabular}

Tables 10 and 11 provide the confusion matrices for the performances of the baseline $\mathrm{CNN}$ and the proposed DnCNN-CNN architecture, respectively, when the German EMODB was mixed with the PCAFETER noise. The CNN exhibits the highest accuracy for boredom (100.0\%), followed by sadness (72.6\%) and anxiety (52.2\%). Disgust, neutral, and anger are recognized as the intended emotions $37.0 \%, 30.4 \%$, and $26.4 \%$ of the time, respectively. Happiness is the least accurately recognized emotion (23.9\%). The highest 
misclassification is observed for the neutral-boredom pair, where neutral is recognized as boredom in $64.6 \%$ of the instances, followed by the happiness-boredom pair $(50.7 \%)$ and the disgust-boredom pair $(45.7 \%)$. The proposed DnCNN-CNN achieves the highest accuracy for boredom (92.6\%), followed by neutral (83.5\%). Sadness, anger, happiness, and anxiety are recognized as the intended emotions in $77.4 \%, 76.0 \%, 67.6 \%$, and $67.2 \%$ of the instances, respectively. Disgust is the least accurately recognized emotion $(65.2 \%)$. The sadness-boredom pair exhibits the highest misclassification, where neutral is recognized as boredom in $21.0 \%$ of the instances, followed by the anxiety-neutral pair, where anxiety is recognized as neutral in $17.9 \%$ of the instances. The proposed DnCNN-CNN (76.6\%) outperforms the baseline $\mathrm{CNN}$ (48.2\%) in the overall accuracy for the EMO-DB mixed with the PCAFETER noise. The DnCNN-CNN also performs better than the baseline CNN for every emotion class except for the boredom emotion. The highest improvement in the classification accuracy is observed for neutral (53.1\%), whereas the lowest improvement in the classification accuracy is observed for sadness (4.8\%).

Table 10. Confusion matrix of the emotion classification results of the baseline CNN that was trained on the German EMO-DB mixed with the PCAFETER noise.

\begin{tabular}{|c|c|c|c|c|c|c|c|c|}
\hline & & \multicolumn{7}{|c|}{ Predicted Emotion } \\
\hline \multirow{8}{*}{ True emotion } & & Neutral & Anger & Boredom & Disgust & Anxiety & Happiness & Sadness \\
\hline & Neutral & $30.4 \%$ & $0.0 \%$ & $64.6 \%$ & $0.0 \%$ & $0.0 \%$ & $0.0 \%$ & $5.1 \%$ \\
\hline & Anger & $3.1 \%$ & $26.4 \%$ & $24.8 \%$ & $7.0 \%$ & $28.7 \%$ & $7.8 \%$ & $2.3 \%$ \\
\hline & Boredom & $0.0 \%$ & $0.0 \%$ & $100.0 \%$ & $0.0 \%$ & $0.0 \%$ & $0.0 \%$ & $0.0 \%$ \\
\hline & Disgust & $2.2 \%$ & $0.0 \%$ & $45.7 \%$ & $37.0 \%$ & $6.5 \%$ & $0.0 \%$ & $8.7 \%$ \\
\hline & Anxiety & $10.4 \%$ & $0.0 \%$ & $19.4 \%$ & $3.0 \%$ & $52.2 \%$ & $0.0 \%$ & $14.9 \%$ \\
\hline & Happiness & $11.3 \%$ & $2.8 \%$ & $50.7 \%$ & $4.2 \%$ & $7.0 \%$ & $23.9 \%$ & $0.0 \%$ \\
\hline & Sadness & $1.6 \%$ & $0.0 \%$ & $25.8 \%$ & $0.0 \%$ & $0.0 \%$ & $0.0 \%$ & $72.6 \%$ \\
\hline \multicolumn{9}{|c|}{ Overall accuracy: $48.2 \%$} \\
\hline
\end{tabular}

Table 11. Confusion matrix of the emotion classification results of the proposed cascaded DnCNN-CNN that was trained on the German EMO-DB mixed with the PCAFETER noise.

\begin{tabular}{|c|c|c|c|c|c|c|c|c|}
\hline & & \multicolumn{7}{|c|}{ Predicted Emotion } \\
\hline \multirow{8}{*}{ True emotion } & & Neutral & Anger & Boredom & Disgust & Anxiety & Happiness & Sadness \\
\hline & Neutral & $83.5 \%$ & $0.0 \%$ & $16.5 \%$ & $0.0 \%$ & $0.0 \%$ & $0.0 \%$ & $0.0 \%$ \\
\hline & Anger & $4.7 \%$ & $76.0 \%$ & $0.8 \%$ & $1.6 \%$ & $3.9 \%$ & $13.2 \%$ & $0.0 \%$ \\
\hline & Boredom & $7.4 \%$ & $0.0 \%$ & $92.6 \%$ & $0.0 \%$ & $0.0 \%$ & $0.0 \%$ & $0.0 \%$ \\
\hline & Disgust & $6.5 \%$ & $0.0 \%$ & $15.2 \%$ & $65.2 \%$ & $8.7 \%$ & $2.2 \%$ & $2.2 \%$ \\
\hline & Anxiety & $17.9 \%$ & $1.5 \%$ & $4.5 \%$ & $1.5 \%$ & $67.2 \%$ & $1.5 \%$ & $6.0 \%$ \\
\hline & Happiness & $12.7 \%$ & $5.6 \%$ & $11.3 \%$ & $1.4 \%$ & $1.4 \%$ & $67.6 \%$ & $0.0 \%$ \\
\hline & Sadness & $1.6 \%$ & $0.0 \%$ & $21.0 \%$ & $0.0 \%$ & $0.0 \%$ & $0.0 \%$ & $77.4 \%$ \\
\hline
\end{tabular}

Tables 12 and 13 provide the confusion matrices for the performance of the baseline CNN and the proposed DnCNN-CNN architecture, respectively, when the German EMODB was mixed with the PSTATION noise. The CNN obtains the highest accuracy for boredom (100.0\%) and the lowest accuracy for anger (10.1\%). Sadness, anxiety, neutral, disgust, and happiness are recognized as the intended emotions in $71.0 \%, 38.8 \%, 24.1 \%$, $23.9 \%$, and $15.5 \%$ of the instances, respectively. The highest misclassification is observed for the neutral-boredom pair, where neutral is recognized as boredom in $70.9 \%$ of the instances. This is followed by the happiness-boredom pair (62.0\%) and the disgust-boredom pair (45.7\%). The DnCNN-CNN achieves the highest classification accuracy for boredom 
(98.8\%) and the lowest classification accuracy for happiness (39.4\%). Sadness (88.7\%) exhibits relatively better accuracies in comparison with disgust $(52.2 \%)$, anxiety $(50.7 \%)$, anger $(46.5 \%)$, and neutral (45.6\%). The highest misclassification is observed for the neutralboredom pair, where happiness is misclassified as boredom $53.2 \%$ of the time. Disgust $(26.1 \%)$, happiness $(29.6 \%)$, and sadness $(11.3 \%)$ are also most frequently misclassified as boredom. Anger and anxiety are most frequently misclassified as neutral $(14.0 \%)$ and sadness $(23.9 \%)$, respectively. The DnCNN-CNN (59.3\%) achieves a higher overall accuracy than the CNN (39.4\%). More specifically, the DnCNN-CNN performs better than the CNN on every emotion class except for boredom.

Table 12. Confusion matrix of the emotion classification results of the baseline CNN that was trained on the German EMO-DB mixed with the PSTATION noise.

\begin{tabular}{|c|c|c|c|c|c|c|c|c|}
\hline & & \multicolumn{7}{|c|}{ Predicted Emotion } \\
\hline \multirow{8}{*}{ True emotion } & & Neutral & Anger & Boredom & Disgust & Anxiety & Happiness & Sadness \\
\hline & Neutral & $24.1 \%$ & $0.0 \%$ & $70.9 \%$ & $0.0 \%$ & $0.0 \%$ & $0.0 \%$ & $5.1 \%$ \\
\hline & Anger & $14.0 \%$ & $10.1 \%$ & $31.8 \%$ & $7.0 \%$ & $30.2 \%$ & $3.9 \%$ & $3.1 \%$ \\
\hline & Boredom & $0.0 \%$ & $0.0 \%$ & $100.0 \%$ & $0.0 \%$ & $0.0 \%$ & $0.0 \%$ & $0.0 \%$ \\
\hline & Disgust & $2.2 \%$ & $0.0 \%$ & $45.7 \%$ & $23.9 \%$ & $8.7 \%$ & $0.0 \%$ & $19.6 \%$ \\
\hline & Anxiety & $13.4 \%$ & $0.0 \%$ & $20.9 \%$ & $1.5 \%$ & $38.8 \%$ & $0.0 \%$ & $25.4 \%$ \\
\hline & Happiness & $9.9 \%$ & $1.4 \%$ & $62.0 \%$ & $4.2 \%$ & $7.0 \%$ & $15.5 \%$ & $0.0 \%$ \\
\hline & Sadness & $0.0 \%$ & $0.0 \%$ & $29.0 \%$ & $0.0 \%$ & $0.0 \%$ & $0.0 \%$ & $71.0 \%$ \\
\hline
\end{tabular}

Table 13. Confusion matrix of the emotion classification results of the proposed cascaded DnCNN-CNN that was trained on the German EMO-DB mixed with the PSTATION noise.

\begin{tabular}{|c|c|c|c|c|c|c|c|c|}
\hline & & \multicolumn{7}{|c|}{ Predicted Emotion } \\
\hline \multirow{8}{*}{ True emotion } & & Neutral & Anger & Boredom & Disgust & Anxiety & Happiness & Sadness \\
\hline & Neutral & $45.6 \%$ & $0.0 \%$ & $53.2 \%$ & $0.0 \%$ & $0.0 \%$ & $0.0 \%$ & $1.3 \%$ \\
\hline & Anger & $14.0 \%$ & $46.5 \%$ & $10.9 \%$ & $7.8 \%$ & $8.5 \%$ & $7.0 \%$ & $5.4 \%$ \\
\hline & Boredom & $0.0 \%$ & $0.0 \%$ & $98.8 \%$ & $0.0 \%$ & $0.0 \%$ & $0.0 \%$ & $1.2 \%$ \\
\hline & Disgust & $10.9 \%$ & $0.0 \%$ & $26.1 \%$ & $52.2 \%$ & $2.2 \%$ & $2.2 \%$ & $6.5 \%$ \\
\hline & Anxiety & $11.9 \%$ & $0.0 \%$ & $13.4 \%$ & $0.0 \%$ & $50.7 \%$ & $0.0 \%$ & $23.9 \%$ \\
\hline & Happiness & $22.5 \%$ & $2.8 \%$ & $29.6 \%$ & $4.2 \%$ & $0.0 \%$ & $39.4 \%$ & $1.4 \%$ \\
\hline & Sadness & $0.0 \%$ & $0.0 \%$ & $11.3 \%$ & $0.0 \%$ & $0.0 \%$ & $0.0 \%$ & $88.7 \%$ \\
\hline
\end{tabular}

Tables 14 and 15 provide the confusion matrices for the performances of the baseline $\mathrm{CNN}$ and the proposed DnCNN-CNN architecture, respectively, when the German EMODB is mixed with the TMETRO noise. The CNN obtains the highest accuracy for boredom $(98.8 \%)$, followed by sadness $(87.1 \%)$, Anxiety, neutral, disgust, and anger have accuracies of $61.2 \%, 57.0 \%, 52.2 \%$, and $38.8 \%$, respectively. Happiness is the least accurately recognized emotion $(28.2 \%)$. The highest misclassification is observed for the neutral-boredom pair, in which neutral is recognized as boredom in 39.2\% of the instances. This is followed by the happiness-boredom pair (38.0\%) and the disgust-boredom pair (28.3\%). The DnCNN$\mathrm{CNN}$ achieves the highest accuracy for boredom (93.8\%), followed by sadness (88.7\%). Neutral $(68.4 \%)$ and anger (68.2\%) exhibit relatively higher classification accuracies than anxiety (59.7\%), happiness (50.7\%), and disgust (50.0\%). Misclassification is the highest for 
the neutral-boredom emotional state pair, where anxiety is misclassified as neutral $30.4 \%$ of the time. This is followed by the happiness-neutral pair, where happiness is misclassified as neutral $28.2 \%$ of the time. The proposed DnCNN-CNN (69.5\%) outperforms the baseline CNN method (58.1\%) in terms of the overall accuracy; more specifically, the DnCNN-CNN performs better than the $\mathrm{CNN}$ on every emotion class except for boredom, disgust, and anxiety.

Table 14. Confusion matrix of the emotion classification results of the baseline CNN that was trained on the German EMO-DB mixed with the TMETRO noise.

\begin{tabular}{|c|c|c|c|c|c|c|c|c|}
\hline & & \multicolumn{7}{|c|}{ Predicted Emotion } \\
\hline \multirow{8}{*}{ True emotion } & & Neutral & Anger & Boredom & Disgust & Anxiety & Happiness & Sadness \\
\hline & Neutral & $57.0 \%$ & $0.0 \%$ & $39.2 \%$ & $0.0 \%$ & $0.0 \%$ & $0.0 \%$ & $3.8 \%$ \\
\hline & Anger & $8.5 \%$ & $38.8 \%$ & $14.0 \%$ & $6.2 \%$ & $22.5 \%$ & $8.5 \%$ & $1.6 \%$ \\
\hline & Boredom & $0.0 \%$ & $0.0 \%$ & $98.8 \%$ & $0.0 \%$ & $0.0 \%$ & $0.0 \%$ & $1.2 \%$ \\
\hline & Disgust & $0.0 \%$ & $0.0 \%$ & $28.3 \%$ & $52.2 \%$ & $13.0 \%$ & $0.0 \%$ & $6.5 \%$ \\
\hline & Anxiety & $9.0 \%$ & $0.0 \%$ & $9.0 \%$ & $1.5 \%$ & $61.2 \%$ & $0.0 \%$ & $19.4 \%$ \\
\hline & Happiness & $16.9 \%$ & $2.8 \%$ & $38.0 \%$ & $4.2 \%$ & $9.9 \%$ & $28.2 \%$ & $0.0 \%$ \\
\hline & Sadness & $1.6 \%$ & $0.0 \%$ & $11.3 \%$ & $0.0 \%$ & $0.0 \%$ & $0.0 \%$ & $87.1 \%$ \\
\hline & & \multicolumn{7}{|c|}{ Overall accuracy: $58.1 \%$} \\
\hline
\end{tabular}

Table 15. Confusion matrix of the emotion classification results of the proposed cascaded DnCNN-CNN that was trained on the German EMO-DB mixed with the TMETRO noise.

\begin{tabular}{|c|c|c|c|c|c|c|c|c|}
\hline & & \multicolumn{7}{|c|}{ Predicted Emotion } \\
\hline \multirow{8}{*}{ True emotion } & & Neutral & Anger & Boredom & Disgust & Anxiety & Happiness & Sadness \\
\hline & Neutral & $68.4 \%$ & $0.0 \%$ & $30.4 \%$ & $0.0 \%$ & $0.0 \%$ & $0.0 \%$ & $1.3 \%$ \\
\hline & Anger & $7.0 \%$ & $68.2 \%$ & $3.9 \%$ & $2.3 \%$ & $6.2 \%$ & $11.6 \%$ & $0.8 \%$ \\
\hline & Boredom & $6.2 \%$ & $0.0 \%$ & $93.8 \%$ & $0.0 \%$ & $0.0 \%$ & $0.0 \%$ & $0.0 \%$ \\
\hline & Disgust & $15.2 \%$ & $2.2 \%$ & $13.0 \%$ & $50.0 \%$ & $8.7 \%$ & $4.3 \%$ & $6.5 \%$ \\
\hline & Anxiety & $23.9 \%$ & $1.5 \%$ & $6.0 \%$ & $0.0 \%$ & $59.7 \%$ & $0.0 \%$ & $9.0 \%$ \\
\hline & Happiness & $28.2 \%$ & $4.2 \%$ & $11.3 \%$ & $1.4 \%$ & $4.2 \%$ & $50.7 \%$ & $0.0 \%$ \\
\hline & Sadness & $1.6 \%$ & $0.0 \%$ & $9.7 \%$ & $0.0 \%$ & $0.0 \%$ & $0.0 \%$ & $88.7 \%$ \\
\hline
\end{tabular}

\section{Discussion}

Recently, CNNs have achieved advanced performance for a wide spectrum of applications. In particular, $\mathrm{CNN}$-based approaches are compelling in terms of recognizing emotions while analyzing recorded speech in a noise-free environment. However, very little attention has been given to their ability to recognize emotional states from noisy speech. Motivated by the effectiveness of the DnCNN for noise reduction in wafer maps and image processing, this study explored their applicability in the speech domain. This was achieved by evaluating the performance of the proposed classifier in the Korean CADKES and German EMO-DB databases under noisy environmental conditions. Specifically, this investigation proposed the DnCNN-CNN architecture, which concatenates the residual-learning-based denoising stage and classification stage. For comparison, this study also analyzed the performance of the baseline CNN. Notably, only a few studies have utilized CNNs for noisy speech emotion recognition. This study provides the first evidence for the feasibility and effectiveness of the DnCNN architecture for boosting the emotion 
recognition performance in speech signals. In addition, this study is the first attempt at using deep learning algorithms to classify Korean emotional speech.

The results show that the proposed DnCNN-CNN achieves a 2-28.4\% increase in the overall accuracy in comparison with the baseline CNN under the PCAFETER, PSTATION, and TMETRO noise conditions. Nonetheless, the two classifiers perform poorly overall on the Korean database that was mixed with the three types of noise. When it was tested with the original CADKES, the proposed DnCNN-CNN exhibited a drastic increase in the overall accuracy. The baseline $\mathrm{CNN}$ also gained a high overall accuracy. These patterns indicate the effect of the noise that is contained in the original Korean CADKES in emotion classification. It also supports the effectiveness of the proposed method in recognizing emotions from speech signals that are corrupted by noise. Furthermore, the performance of the proposed method on the German EMO-DB highlights the potential efficiency of the DnCNN-CNN for recognizing emotions from noisy speech. Meanwhile, it exhibits higher accuracies than the CNN under the three noise conditions. The results of the Bayesian Wilcoxon Signed Rank test based on [54] show that the probability of higher accuracy of the proposed DnCNN-CNN than the baseline CNN is $100 \%$.

The overall DnCNN results are conceptually consistent with those of previous research on the noise reduction of image sequences [41] and semiconductor wafer maps [42] in terms of its high potential in denoising tasks across domains. The baseline-CNN-based results are also largely consistent with those of previous studies [24,32-39]. Finally, the high accuracy of speech emotion recognition for the original Korean CADKES demonstrates the effectiveness and versatility of CNNs in a variety of recognition tasks.

However, there are some important limitations to this research. First, the performance of the proposed algorithm in emotion recognition tasks for noisy speech was tested in only two languages. It remains undetermined whether the proposed DnCNN is effective in a language-universal manner. Second, the effectiveness of the DnCNN in noisy speech emotion recognition is limited because its performance varies remarkably as a function of the noise level. In particular, the DnCNN performs drastically better on the original CADKES than the CADKES mixed with different types of noise. Further studies are required to verify the effectiveness of the proposed DnCNN-CNN on noisy speech emotion recognition tasks.

As the proposed algorithm performed considerably better when using German speech than when using Korean speech, one possible direction for future studies is to boost the denoising performance and robustness of the $\mathrm{DnCNN}-\mathrm{CNN}$ on emotional speech signals to which heavy noise is added. Another future research direction is to corroborate the applicability of the proposed method by exploring whether the proposed DnCNN-CNN performs well in enhancing the recognition of noisy emotional speech in languages other than Korean and German.

Author Contributions: Conceptualization and design, Y.N.; creation of the Korean emotional speech dataset and writing, Y.N.; supervision, C.L. Both authors have read and agreed to the published version of the manuscript. All authors have read and agreed to the published version of the manuscript.

Funding: This work was funded by a National Research Foundation of Korea (NRF) grant funded by the Korean government (No. 2017S1A6A3A01078538).

Institutional Review Board Statement: The recording was carried out in accordance with relevant guidelines and regulations, and the recording methods of the database were approved by the ChungAng University Institutional Review Board, Republic of Korea. Informed written consent was obtained prior to recording from all participants.

Informed Consent Statement: Informed consent was obtained from all participants involved in the study.

Data Availability Statement: The datasets generated and/or analyzed during the present study are available from the first author or the corresponding author on reasonable request. 
Acknowledgments: We thank Sungeu Chae for his assistance in implementing the DnCNN-CNN methodology and for his helpful discussions on this manuscript.

Conflicts of Interest: The authors declare no conflict of interest. The funders had no role in the design of the study; in the collection, analyses, or interpretation of data; in the writing of the manuscript; or in the decision to publish the results.

\section{References}

1. Anagnostopoulos, C.-N.; Iliou, T.; Giannoukos, I. Features and classifiers for emotion recognition from speech: A survey from 2000 to 2011. Artif. Intell. Rev. 2012, 43, 155-177. [CrossRef]

2. Vidrascu, L.; Devillers, L. Real-Life Emotion Representation and Detection in Call Centers Data. In Proceedings of the Transactions on Petri Nets and Other Models of Concurrency XV, Computing and Intelligent Interaction; First International Conference, ACII 2005, Beijing, China, 22-24 October 2005; Springer Science and Business Media LLC: Berlin/Heidelberg, Germany, 2005; Volume 3784, pp. 739-746.

3. López-De-Ipiña, K.; Alonso-Hernández, J.; Solé-Casals, J.; Travieso-González, C.; Ezeiza, A.; Faundez-Zanuy, M.; Calvo, P.; Beitia, B. Feature selection for automatic analysis of emotional response based on nonlinear speech modeling suitable for diagnosis of Alzheimer's disease. Neurocomputing 2015, 150, 392-401. [CrossRef]

4. Hansen, J.H.; Cairns, D.A. ICARUS: Source generator based real-time recognition of speech in noisy stressful and Lombard effect environments. Speech Commun. 1995, 16, 391-422. [CrossRef]

5. Tickle, A.; Raghu, S.; Elshaw, M. Emotional recognition from the speech signal for a virtual education agent. J. Phys. Conf. Ser. 2013, 450, 012053. [CrossRef]

6. Tarng, W.; Chen, Y.Y.; Li, C.L.; Hsie, K.R.; Chen, M. Applications of support vector machines on smart phone systems for emotional speech recognition. World Acad. Sci. Eng. Technol. 2010, 72, 106-113.

7. Jassim, W.; Paramesran, R.; Harte, N. Speech emotion classification using combined neurogram and INTERSPEECH 2010 paralinguistic challenge features. IET Signal. Process. 2017, 11, 587-595. [CrossRef]

8. Vayrynen, E.; Kortelainen, J.; Seppanen, T. Classifier-based learning of nonlinear feature manifold for visualization of emotional speech prosody. IEEE Trans. Affect. Comput. 2012, 4, 47-56. [CrossRef]

9. Gobl, C.; Ní Chasaide, A. The role of voice quality in communicating emotion, mood and attitude. Speech Commun. 2003, 40, 189-212. [CrossRef]

10. Deb, S.; Dandapat, S. Emotion Classification Using Segmentation of Vowel-Like and Non-Vowel-Like Regions. IEEE Trans. Affect. Comput. 2019, 10, 360-373. [CrossRef]

11. Zhou, G.; Hansen, J.; Kaiser, J. Nonlinear feature based classification of speech under stress. IEEE Trans. Speech Audio Process. 2001, 9, 201-216. [CrossRef]

12. Vrysis, L.; Hadjileontiadis, L.; Thoidis, I.; Dimoulas, C.; Papanikolaou, G. Enhanced Temporal Feature Integration in Audio Semantics via Alpha-Stable Modeling. J. Audio Eng. Soc. 2021, 69, 227-237. [CrossRef]

13. Lin, Y.-L.; Wei, G. Speech emotion recognition based on HMM and SVM. In Proceedings of the 2005 International Conference on Machine Learning and Cybernetics, Guangzhou, China, 18-21 August 2005; Volume 8, p. 4898.

14. Schuller, B.; Rigoll, G.; Lang, M. Speech emotion recognition combining acoustic features and linguistic information in a hybrid support vector machine-belief network architecture. In Proceedings of the 2004 IEEE International Conference on Acoustics, Speech, and Signal Processing, Montreal, QC, Canada, 17-21 May 2004; Volume 1, p. 577.

15. Chauhan, A.; Koolagudi, S.G.; Kafley, S.; Rao, K.S. Emotion recognition using LP residual. 2010 IEEE Stud. Technol. Symp. (TechSym) 2010, 255-261. [CrossRef]

16. Ingale, B.A.; Chaudhari, D. Speech emotion recognition using hidden Markov model and support vector machine. Int. J. Soft Comput. Eng. 2012, 2, 235-238.

17. Shaw, A.; Kumar, R.; Saxena, S. Emotion Recognition and Classification in Speech using Artificial Neural Networks. Int. J. Comput. Appl. 2016, 145, 5-9. [CrossRef]

18. Bhatti, M.W.; Wang, Y.; Guan, L. A neural network approach for human emotion recognition in speech. In Proceedings of the 2004 IEEE International Symposium on Circuits and Systems (IEEE Cat. No.04CH37512), Vancouver, BC, Canada, 23-26 May 2004; pp. 181-184.

19. Lee, C.-C.; Mower, E.; Busso, C.; Lee, S.; Narayanan, S. Emotion recognition using a hierarchical binary decision tree approach Speech Commun. 2011, 53, 1162-1171. [CrossRef]

20. Dahl, G.E.; Yu, D.; Deng, L.; Acero, A. Context-Dependent Pre-Trained Deep Neural Networks for Large-Vocabulary Speech Recognition. IEEE Trans. Audio Speech Lang. Process. 2012, 20, 30-42. [CrossRef]

21. Palaz, D.; Magimai-Doss, M.; Collobert, R. Analysis of CNN-based Speech Recognition System Using Raw Speech as Input. In Proceedings of the Annual Conference of the International Speech Communication Association, INTERSPEECH, Dresden, Germany, 6-10 September 2015; pp. 11-15.

22. Simonyan, K.; Zisserman, A. Very Deep Convolutional Networks for Large-scale Image Recognition. In Proceedings of the 3rd International Conference on Learning Representations, ICLR 2015, San Diego, CA, USA, 7-9 May 2015; Conference Track Proceedings. 
23. Goldberg, Y. A Primer on Neural Network Models for Natural Language Processing. J. Artif. Intell. Res. 2016, 57, 345-420. [CrossRef]

24. Badshah, A.M.; Ahmad, J.; Rahim, N.; Baik, S.W. Speech Emotion Recognition from Spectrograms with Deep Convolutional Neural Network. In Proceedings of the 2017 International Conference on Platform Technology and Service (PlatCon), Busan, Korea, 13-15 February 2017; pp. 1-5.

25. Chen, L.; Mao, X.; Xue, Y.; Cheng, L.L. Speech emotion recognition: Features and classification models. Digit. Signal. Process. 2012, 22, 1154-1160. [CrossRef]

26. Lim, W.; Jang, D.; Lee, T. Speech emotion recognition using convolutional and Recurrent Neural Networks. In Proceedings of the 2016 Asia-Pacific Signal and Information Processing Association Annual Summit and Conference (APSIPA), Jeju, Korea, 13-16 December 2016; pp. 1-4.

27. Bengio, Y.; Courville, A.; Vincent, P. Representation Learning: A Review and New Perspectives. IEEE Trans. Pattern Anal. Mach. Intell. 2013, 35, 1798-1828. [CrossRef]

28. Han, K.; Yu, D.; Tashev, I. Speech Emotion Recognition Using Deep Neural Network and Extreme Learning Machine. In Proceedings of the Annual Conference of the International Speech Communication Association, INTERSPEECH, Singapore, 14-18 September 2014; pp. 223-227.

29. Jiang, W.; Wang, Z.; Jin, J.S.; Han, X.; Li, C. Speech Emotion Recognition with Heterogeneous Feature Unification of Deep Neural Network. Sensors 2019, 19, 2730. [CrossRef] [PubMed]

30. Lee, J.; Tashev, I. High-level Feature Representation Using Recurrent Neural Network for Speech Emotion Recognition. In Proceedings of the Annual Conference of the International Speech Communication Association, INTERSPEECH, Dresden, Germany, 6-10 September 2015; pp. 1537-1540.

31. LeCun, Y.; Bengio, Y.; Hinton, G. Deep learning. Nature 2015, 521, 436-444. [CrossRef]

32. Schluter, J.; Böck, S. Improved musical onset detection with Convolutional Neural Networks. In Proceedings of the 2014 IEEE International Conference on Acoustics, Speech and Signal Processing (ICASSP), Florence, Italy, 4-9 May 2014; pp. 6979-6983.

33. Abdel-Hamid, O.; Mohamed, A.; Jiang, H.; Deng, L.; Penn, G.; Yu, D. Convolutional neural networks for speech recognition. IEEE Trans. Audio Speech Lang. Process. 2014, 22, 1533-1545. [CrossRef]

34. Vrysis, L.; Tsipas, N.; Thoidis, I.; Dimoulas, C. 1D/2D Deep CNNs vås. Temporal Feature Integration for General Audio Classification. J. Audio Eng. Soc. 2020, 68, 66-77. [CrossRef]

35. Parthasarathy, S.; Tashev, I. Convolutional Neural Network Techniques for Speech Emotion Recognition. In Proceedings of the 2018 16th International Workshop on Acoustic Signal Enhancement (IWAENC), Dhaka, Bangladesh, 28-30 November 2019; pp. 121-125.

36. Zhao, J.; Mao, X.; Chen, L. Learning deep features to recognise speech emotion using merged deep CNN. IET Signal. Process. 2018, 12, 713-721. [CrossRef]

37. Yenigalla, P.; Kumar, A.; Tripathi, S.; Singh, C.; Kar, S.; Vepa, J. Speech Emotion Recognition Using Spectrogram and Phoneme Embedding. In Proceedings of the Interspeech 2018, International Speech Communication Association, Hyderabad, India, 2-6 September 2018; pp. 3688-3692.

38. Anvarjon, T.; Kwon, S. Deep-Net: A Lightweight CNN-Based Speech Emotion Recognition System Using Deep Frequency Features. Sensors 2020, 20, 5212. [CrossRef]

39. Vryzas, N.; Vrysis, L.; Matsiola, M.; Kotsakis, R.; Dimoulas, C.; Kalliris, G. Continuous Speech Emotion Recognition with Convolutional Neural Networks. J. Audio Eng. Soc. 2020, 68, 14-24. [CrossRef]

40. Abbaschian, B.; Sierra-Sosa, D.; Elmaghraby, A. Deep Learning Techniques for Speech Emotion Recognition, from Databases to Models. Sensors 2021, 21, 1249. [CrossRef] [PubMed]

41. He, K.; Zhang, X.; Ren, S.; Sun, J. Deep residual learning for image recognition. In Proceedings of the 2016 IEEE Conference on Computer Vision and Pattern Recognition, Las Vegas, NV, USA, 27-30 June 2016; pp. 770-778.

42. Chae, S.G.; Bae, S.J. Cascaded Convolutional Neural Network for Wafer Bin Map Defect Pattern Classification. In Proceedings of the Informs Annual Meeting 2019 WA32, Washington, DC, USA, 20-23 October 2019.

43. Mustafa, M.B.; Yusoof, M.A.M.; Don, Z.M.; Malekzadeh, M. Speech emotion recognition research: An analysis of research focus. Int. J. Speech Technol. 2018, 21, 137-156. [CrossRef]

44. Burkhardt, F.; Paeschke, A.; Rolfes, M.; Sendlmeier, W.; Weiss, B. A Database of German Emotional Speech. In Proceedings of the Annual Conference of the International Speech Communication Association, INTERSPEECH 2005, Lisbon, Portugal, 4-8 September 2005; pp. 1517-1520.

45. Boersma, P.; Weenink, D. Praat: Doing Phonetics by Computer [Computer Program]. Version 6.0.37. Available online: http: / / www.praat.org/ (accessed on 28 June 2020).

46. Thiemann, J.; Ito, N.; Vincent, E. The Diverse Environments Multi-channel Acoustic Noise Database (DEMAND): A database of multichannel environmental noise recordings. In Proceedings of the Meetings on Acoustics ICA2013, Montreal, QC, Canada, 2-7 June 2013; p. 3591.

47. Badshah, A.M.; Rahim, N.; Ullah, N.; Ahmad, J.; Muhammad, K.; Lee, M.Y.; Kwon, S.; Baik, S.W. Deep features-based speech emotion recognition for smart affective services. Multimed. Tools Appl. 2019, 78, 5571-5589. [CrossRef] 
48. Trigeorgis, G.; Ringeval, F.; Brueckner, R.; Marchi, E.; Nicolaou, M.A.; Schuller, B.; Zafeiriou, S. Adieu features? End-to-end speech emotion recognition using a deep convolutional recurrent network. In Proceedings of the 2016 IEEE International Conference on Acoustics, Speech and Signal Processing (ICASSP), Shanghai, China, 20-25 March 2016; pp. 5200-5204.

49. Xue, F.-F.; Peng, J.; Wang, R.; Zhang, Q.; Zheng, W.-S. Improving Robustness of Medical Image Diagnosis with Denoising Convolutional Neural Networks. In Proceedings of the International Conference on Medical Image Computing and ComputerAssisted Intervention, Shenzhen, China, 13-17 October 2019; pp. 846-854.

50. Zhang, K.; Zuo, W.; Chen, Y.; Meng, D.; Zhang, L. Beyond a Gaussian Denoiser: Residual Learning of Deep CNN for Image Denoising. IEEE Trans. Image Process. 2017, 26, 3142-3155. [CrossRef]

51. Kim, J.; Lee, J.K.; Lee, K.M. Accurate image super-resolution using very deep convolutional networks. In Proceedings of the IEEE Conference on Computer Vision and Pattern Recognition, Las Vegas, NV, USA, 27-30 June 2016; pp. 1646-1654.

52. Kingma, D.P.; Ba, J. Adam: A method for stochastic optimization. In Proceedings of the International Conference Learn Represent. (ICLR), San Diego, CA, USA, 5-8 May 2015.

53. Ioffe, S.; Szegedy, C. Batch normalization: Accelerating deep network training by reducing internal covariate shift. In Proceedings of the 32nd International Conference on Machine Learning, ICML, Lille, France, 6-11 July 2015; pp. 448-456.

54. Benavoli, A.; Corani, G.; Demšar, J.; Zaffalon, M. Time for a change: A tutorial for comparing multiple classifiers through Bayesian analysis. J. Mach. Learn. Res. 2017, 18, 2653-2688. 